\title{
Managing Pharmacy Risk in Physician Groups
}

\author{
Successfully negotiating and working under pharmacy risk-sharing \\ contracts with managed care organizations requires physicians to \\ improve their pharmacy management expertise.
}

\section{Linda DeLaet}

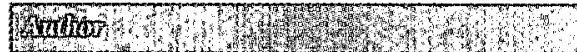

LINDA DELAET, Pharm.D., is Director of Pharmacy, Monarch HealthCare, Mission Viejo, CA.

AUTHOR CORRESPONDENCE: Linda DeLaet, Pharm.D., Director of Pharmacy, Monarch HealthCare, 25950 Acero, Suite 200, Mission Viejo, CA 92691.

Copyright@ 1999, Academy of Managed Care Pharmacy, Inc. All rights reserved.
$\mathrm{D}$ uring the last 15 years, the number and type of managed care contracts has grown appreciably. As health plans have struggled to meet the mandate to control costs, they have revamped old tools and applied them in new ways. One such technique involves pharmacy cost risk sharing with physician groups. Pharmacy risk sharing invites all parties to manage the pharmacy benefit jointly and share the positive or negative financial consequences. Long used in California, risk sharing is becoming more common in other parts of the country and has presented a new opportunity for pharmacists to apply their clinical and administrative skills.

In risk-sharing agreements, the managed care organization (MCO) budgets a fixed amount for pharmaceuticals. Under such contracts, the MCO agrees to share any savings with the physician group or split cost overages associated with prescription drugs. The proportion of the split varies, but 50/50 agreements are common. Any special circumstances or exceptions must be specified in the contract.

MCOs offer physician groups risksharing agreements for many reasons. When contracting with physician groups, health plans exercise less control than they do in more structured health maintenance organizations. Providers in physician groups retain significantly more autonomy. Health plans find that policies and control tools used in more traditional managed care arrangements are harder to enforce, and other means are needed to ensure that cost is still a consideration.

On the surface, pharmacy risk may appear fairly straightforward, but it is considerably more complex than it appears to the novice. Some physician groups have been successful at sharing risk, but the landscape is littered with disasters as well. Patrick Kapsner, CEO of Bristol Medical Group, says that a movement is afoot in California to limit sharing of pharmacy risk or prohibit it entirely. According to Kapsner, providers cannot control many of the factors built into pharmacy risk and therefore cannot manage it. The lack of pharmacy expertise and the dearth of good management tools are the biggest issues for providers.

\section{RISING PHARMACY COSTS}

The pharmacy benefit is particularly important right now, as the cost of drugs averages about $\$ 16$ per member per month (PMPM) in commercial populations and \$53 PMPM in senior populations. Pharmacy costs are expected to rise $12 \%-16 \%$ in the next three to four years. Many factors have contributed to increased drug costs, including:

$\boldsymbol{\Delta}$ a change in the mix of drugs being prescribed, such as the shift from $\mathrm{H} 2$ receptor blockers to proton pump inhibitors with higher acquisition costs;

$\boldsymbol{\Delta}$ an increasing number of new drugs approved by the FDA;

$\Delta$ changes in manufacturers' pricing practices that in some cases increased acquisition cost of generic drugs;

$\boldsymbol{\Delta}$ an aging American population that has increased the demand for prescription medication;

$\Delta$ new drug technologies which offer costly options for many disease states;

$\Delta$ more medications to treat illnesses 
that previously had no ameliorative or curative options, and more medications replacing surgery; and

$\Delta$ the enrichment of pharmacy benefits to MCO members, such as changes in copayments and benefit maximums.

Direct-to-consumer advertising, at an estimated cost of $\$ 1.3$ billion annually, also has changed the drug market quickly and permanently. Advertising in the U.S is a sophisticated and influential process, and adaptation of prescription drug advertising to end-point consumers has complicated the provider-patient relationship. Patients may appear at appointments with preconceived ideas about their problems and the medications that should offer relief. Physicians report pressure to prescribe a drug that is brand specific at a greater cost. Add increased Internet access to this mixture, and the health care consumer is an informed (and sometimes misinformed) participant in the health care process. The Wall Street Journal reports that $32 \%$ of the $47 \mathrm{mil}-$ lion Americans on the Internet are accessing health care information.

Health plans are under tremendous pressure and growing scrutiny due to media coverage of managed care issues. Regulatory agencies have begun to express concern about possible consumer inconvenience resulting from health plan mergers and acquisitions. Actual or perceived consumer dissatisfaction with the pharmacy benefit has increased scrutiny of formularies and medication issues. Regulatory oversight from the California Department of Corporations has made aggressive formulary management, prior authorization, and other tools less attractive to health plans in that state. The combination of rising costs and increased regulatory pressures has led many California MCOs to turn to risk sharing to help control pharmacy costs.

\section{CONTRACTUAL AGREEMENTS}

The contract covering the pharmacy benefit is, by virtue of the various factors that influence it, extremely complex. While much of the contract language is boilerplate, physician groups must be cautious when negotiating a new contract. Among the most serious mistakes a physician group can make in pharmacy risk-sharing agreements are:

$\boldsymbol{\Delta}$ Taking risk without evaluating the environment. Assessment of the environment requires appropriate data on use, costs, and problems from past years.

A Accepting risk without an eye toward managing it. Managing pharmacy risk requires experience, investment, and excellent communication. Pharmacy risk will not manage itself, and moderate efforts may be unsuccessful.

$\boldsymbol{\Delta}$ Ignoring the group's long-term interest. Every contract should have provisions for changes that may occur in the health plan or pharmacy benefit. Elements that may change include addition of new drugs, extent of coverage, or changes to the copay amount.

The best contract is negotiated with an understanding that all parties must be positioned to win; if parties perceive themselves as adversaries, risk sharing will fail. The goals must be the same for everyone, and all must recognize that good risk-sharing agreements encourage the best care for patients at the lowest cost; make prescribers partners in the medical, legal, and financial aspects of care; and require clear, honest communication of all important historical information.

When all parties are invested in optimal cost-effective management, the chances that profit will be shared are greater than the chances that losses will be divided.

\section{MANAGEMENT MECHANISMS}

Mechanisms used to manage risk are not new or innovative. For the most part, they are the tried-and-true tools of managed care adapted to a challenging milieu. Physician groups may have to deal with large numbers of health plans and physicians. Most deal with multiple health plan formularies.

Formulary management in physician groups is not as straightforward as it can be in more controlled environments. The trend is moving toward use of preferred drug lists for those categories of drugs that are most costly and used most frequently. In general, physician groups find that focusing on 10 or fewer drug categories is simpler for prescribers to remember and apply, allowing cost savings to be accomplished and sustained. For example, many plans focus their efforts on ACE inhibitors, gastrointestinal drugs, lipid-lowering agents, antibiotics, and antidepressants. Including larger numbers of drugs and less-used drug categories results in only marginal gain in cost savings and may hinder progress in controlling expenditures in the costly categories.

Physician education is a key element to success. To succeed in pharmacy risksharing agreements, physician education should include:

A specific details regarding the group's risk arrangement;

A peer review or report card feedback;

$\Delta$ academic detailing;

A disease state management programs; and

$\Delta$ physician-patient interaction.

Physicians must understand their role as important organizational stakeholders. In many groups, physicians own part or all of the association; failure to work toward mutual goals will affect the bottom line. Physicians will understand that their prescribing practices will be reflected in key quality and utilization measures of the care they provide. This, in turn, may affect the endof-the-year financial status of the group and of themselves individually.

Groups can help their physicians by providing information about individual prescribing profiles and by providing comparative data. Explaining how physicians can benefit by reviewing the data helps open doors to cooperation. Experts tend to agree that the information should be concise, factual, and delivered as promptly as possible to effect the most change. Types of information can include the prescriber's top 10 drugs, suggestions for change, explanation of generic or therapeutic equivalence, and comparison with other physicians in the prescriber's specialty. 
Academic detailing, or personal visits by pharmacists to physicians to discuss preferred agent or present balanced information about a drug or drug class, can be useful. This type of visit should be short and to the point. Some organizations offer continuing education units, which serve as an enticement for physician participation. Academic detailing can help physicians understand advertising weaknesses, identify cost-effective alternatives, dispel misconceptions, and notify physicians of generic availability.

The rise of clinical guidelines has been helpful. Independent, unbiased groups have created disease-specific guidelines to help clinicians identify best practices. Disease state management, as opposed to a focus on a particular drug or category of drugs, helps physicians consider all appropriate factors before planning care. However, the guidelines are often lengthy and difficult to follow. When possible, reducing the key points to a concise list or flow sheet allows clinicians to apply the guideline more frequently.

Training on communication techniques is important for all direct-care providers, especially now that patients are the direct targets of pharmaceutical advertising. Physicians need help in learning to communicate with patients whose opinions are already prejudiced by advertising, lay press articles, or hearsay when they appear for treatment. This type of communication is very much like academic detailing, and physicians must learn to present factual information in a convincing manner

In general, all educational or informational offerings should be brief and focused. Physicians receive quantities of mail and generally respond best to short, eye-catching materials. Innovation is important. Use of facsimile or electronic mail broadcasts is an efficient way to circulate information.

\section{CONSTANT CHANGE}

Like all business practices, risk sharing changes constantly. Physician groups have to stay abreast of clinical, regulatory, and financial changes and to plan well in advance. Assessing the manage- ment tools currently being used in risksharing agreements can suggest areas for improvement. For example, the tools discussed above have some imperfections that make them difficult to use:

$\Delta$ Academic detailing requires welltrained pharmacists who visit physicians often. They must compete with pharmaceutical sales representatives who usually have considerably more resources than group-employed pharmacists. Thus, academic detailing can be very time consuming and costly.

$\Delta$ Most of the tools described above are provided before or after, but rarely during, patient visits. Point-of-service information would be more effective.

$\Delta$ Communication initiatives compete with information from many other sources. Unless the group plans and implements its communications well, its messages may be lost in a fruitless effort.

Several new developments may help physician groups direct their efforts for maximum effectiveness. First, data management has been physician groups' nemesis. Different plans employ different software, some insurers have changed formats frequently, data have not been shared readily, and consolidation and interpretation of data have been difficult. The combination of newer software programs and experience working together has helped plans and physician groups clarify expectations and share data more easily, but intelligence and resources are still needed to improve performance.

Second, use of electronic medical record (EMR) systems offers promise. Point-of-service intervention and communication is its strength. As the electronic record becomes the norm rather than the exception, pharmacists can expect to see online prescribing. With this advent will come prescriber prompting; as physicians prescribe medications, an automatic prompt will suggest more costeffective alternatives or refer the prescriber to online disease state management information. EMR systems also provide quick Internet access and improve management of multiple formularies.

Finally, member education and involvement will be necessary for optimal cost savings. Health plans and groups will have to include members in the equation and thus provide more member information in appropriate formats. One successful program involves providing an information sheet on the issue of forgoing an antibiotic when a viral infection is diagnosed. These sheets, based on Centers for Disease Control and Prevention guidelines, help physicians explain why they are not prescribing an antibiotic and help members understand the process. Other teaching aids can focus on proper use of medication and preventive health care.

Pharmacy risk sharing is complex and can have significant financial consequences if not properly managed. Physician groups and health plans should structure win-win agreements to achieve best results. Good data and communication are critical to the negotiations. Physician groups need to have a clear understanding of pharmacy risk language in the contract, and they must be prepared to manage that risk.

Traditional principles of pharmacy management must be applied in new and innovative ways within physician groups. "We, as physicians, want to do what is best for our patients," says Monarch HealthCare Medical Group Family Practice physician Bruce Van Vranken. "We go to continuing medical education seminars to learn the latest treatments to help improve the quality and quantity of our patients' lives. An explosion of medical information confronts [us]on a daily basis. Education of the physician using modern communication systems is key to providing stateof-the-art, yet affordable, medical care. Improved communication systems with the physician group's pharmacy specialist, using modern communication devices such as electronic medical records, will be a critical part of any physician's ongoing practice. The pharmacist is a key member of the health care team in providing the best possible care through effective pharmaceutical utilization." 